\title{
Performance Comparison of HS-TCP and TCP in Hierarchical Mobile IPv6 Network
}

\author{
Nur Hayati ${ }^{* 1}$, Mahesa Adhitya Putra ${ }^{2}$ \\ ${ }^{1}$ Department of electrical Engineering, Faculty of Engineering, Universitas Muhammadiyah Yogyakarta \\ Address: Jl. Lingkar Selatan, Kasihan, Bantul, Daerah Istimewa Yogyakarta, 55183 Phone: (0274) 387656 \\ ${ }^{2}$ Department of electrical Engineering, Faculty of Engineering, Universitas Indonesia \\ Address: Pondok Cina, Beji, Depok, West java. 16424 Phone : (021) 7867222 \\ *Corresponding author, e-mail: nha.nurhayati@gmail.com ${ }^{1}$; mahesa.adhitya@ gmail.com ${ }^{2}$
}

\begin{abstract}
Technology information has been growing rapidly in the last few years. HMIPv6 is one of environment used in mobility internet connection. One of transmission used in this method is Transmission Control Protocol (TCP). TCP has a normal connection that connect mobile node to the internet and has a normal speed of transmission. This paper tries to compare TCP with another protocol. High Speed Transmission Control Protocol (HS-TCP) is a transport protocol that introduces new method by improving general algorithm of TCP in reducing time of loss recovery. HS$T C P$ is compared with $T C \neg P$ to see the performance of each protocol. The simulation of these protocols is using Network Simulator 2.31 (NS-2.31). The topology of HMIPv6 concludes 1 Home Agent (HA), 2 Foreign Agent (FA), 1 Mobile Node (MN), 1 Correspondent Node $(C N)$. The performance measurement is processed when MN moved from FA2 to FA1 while communicating with CN. Throughput of HMIPv6 will become the parameters for QoS. The result of the simulation shows that HS-TCP has a better performance than TCP by looking at the throughput which ran on the HMIPv6 topology. This simulation have proved that HS-TCP are suitable to be implemented in

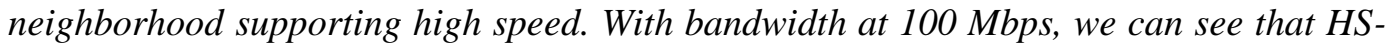
TCP is $32 \%$ better than HS-TCP, in 500 Mbps HS-TCP is better at $96 \%$ than TCP. In 1Gbps, HS-TCP is better at $85 \%$ than TCP and in 10Gbps, HS-TCP is better at $86 \%$ than TCP.
\end{abstract}

Keywords: HMIPv6, TCP, HS-TCP, NS-2.31, QoS

\section{Introduction}

Nowadays, information technology growing rapidly which is developed diligently by many companies. The more mobile device will make it easier to communicate with other devices. This technology is getting more popular and most people will choose devices that support mobility in multimedia. Due to high demand of mobile services that involve internet protocol connection in any kind of application, addressing scheme use IP version 4 has been replaced by IP version 6 . Mobility is really needed in real-time communications such as chatting, video conference,
Voice over IP and so on. As time goes by, this condition will affect the used of protocol in mobile network. Protocols must be improved to fulfill the need of data transmission; the faster is the better, especially for transmit high quality data. Bo $\mathrm{Hu}$ at al in [1] explained that some protocol related to mobility management are important to be develop. On the other hand, a certain method to increase the speed could be done by rearrange node hierarchy. As explained by Soliman et all in [2] that management for hierarchical mobility in IP version 6 network are made to reduce the number of signalling among mobile node. This design has nodes served to increase the handover speed. Meanwhile Zulkefleee et all in [3] said that 
hierarchical model proposed in mobile network are able to speed up local mobility.

Transmission Control Protocol (TCP) is usually linked together with Internet Protocol (IP) since both of the above services are complementary. These protocols are responsible for carrying out the internet's basic operations. The sending of information online is facilitated by TCP as it breaks down data into packets for quicker transmission.

There is a protocol that has a better performance from a TCP in large congestion windows. The HSTCP, an update of TCP that reacts better when using a large congestion windows on highbandwidth, high-latency networks. This paper discusses about the integration of High Speed Transmission Control Protocol (HS-TCP) method in Hierarchical Mobile Internet Protocol version Six (HMIPv6).

As mentioned before, the study and the experimental test depend on the analysis of he behavior of two TCP's over HMIPv6 network topology. There are some terminology that supports mobility in IPv6 as explained in [3], namely Home Agent (HA), Mobile Node (MN), Correspondent Node (CN), Mobility Anchor Point (MAP) and some others. Mobile Internet IPv6 allows transparent routing of IPv6 packets to MN (Mobile Node). Home Agent will receive all packet delivered to mobile node in a triangular routing. Firstly, packet route to home subnet of mobile node, and next it will be forwaded to outside network where of mobile node stay. Meanwhile, communication built from mobile node to its correspondent are direct communication although it does not same way when correspondent node response to mobile node. Correspondent node need home agent to relay its message to mobile node or it can be say that is indirect communication. Binding updates are send every time when mobile node move to outside network (foreign agent). Home agent and all correspondent node should receive this message in order to carry on communication

The main concept of HMIPv6 is implement mobility anchor point to handle local handover so that HMIPv6 could performs well in micro handoff although it shows insufficient when comes to macro handoff.

In designing this simulation, there are some limitations to the problem, and then it can be determine how far this simulation can presents the objective. Firstly, a Hierarchical Mobile IPv6 topology will be created in the network. This Hierarchical Mobile IPv6 use TCP as a protocol to transfer data from one network to another network. This limitation was created according to a simple network; the limit scope is in intra domain ability. For the mobility, it used horizontal handover from an access point to another access point.

The other limitation of this simulation is using throughput as parameter to see the performance of the network, how is the work of a TCP and HSTCP. The result of this both protocol in Hierarchical Mobile IPv6 will be explained later.

Communication is available in IPv6 because there is support that called Mobile IPv6, so communication in $\mathrm{MN}$ with host that connected in internet is available, though $\mathrm{MN}$ is far away from Home Network. When MN moves from a network to another network, Home Agent has a duty to register MN to Foreign Agent. So MN can still manage the connection though $\mathrm{MN}$ is not in the Home Network.

The rest of this paper is organized as follows. The first section presents an introduction to this paper. Then the second section presents information about an overview of TCP's and HMIPV6 systems. Researchers proposed topology and simulation in Section III, while section IV presents the simulation scenario and the results. This paper is concluded in Section V.

\section{Overview of HMIPv6 SYSTEMS, TCP and HS-TCP}

\section{II.1. Hierarchical Mobile IPv6}

A Hierarchical Mobile IPv6 (HMIPv6) was designed to enhanced mobility problem in MIPv6. The key concept behind HMIPv6 is to locally handle handovers by the usage of an entity called Mobility Anchor Point (MAP) located at any level in a hierarchical network of routers to support micromobility management. Hierarchical mobility management for Mobile IPv6 is designed to reduce the amount of signalling between the mobile node, its correspondent nodes, and its home agent. The 
Mobility Anchor Point (MAP) is used to improve the performance of Mobile IPv6 in terms of handover speed.

Geneal problem in mobile network could not be solved by MIPv6 as explained by Z. Kusini et al in [4]. Either local or hierarchical network are difficult to be analyzed by MIPv6 protocol. Therefore to overcome the shortcoming of non local mobility in MIPv6, a hierarchical scheme divide in to micro and macro mobility. Soliman et al. in [2] proposed Mobility Anchor Point (MAP) as a new node in MIPv6 network. As be shown in Fig.1. there is a node in outside home network but it act like local. This MAP router could be put at any tier in a HMIP network, that it could be categorized as a micromobility.

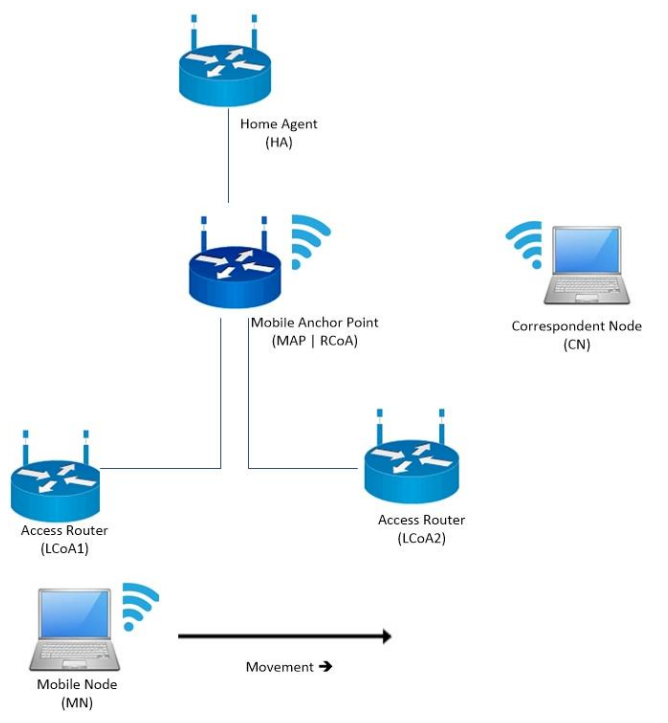

Fig. 1. HMIPv6 Topology

HMIPv6 could be implement when mobile node has the ablitity to dectect HMIPv6 environment while MAP discovery has to choose the setting of hierarchical network. Z. Kusin and M.S. Zakaria describe in [4] that mobile node nee to configure two kind of address namely a regional care of address (RCoA) and an on link care of address (LCoA) in order to use mobility anchor point router in HMIPv6. Access router (AR) send local address to mobile node as its LCoA. While RCoA could be received when mobile node go to outside network. Regional care of address delivered during MAP discover mobile node using Router Advertisement (RA). Mobility anchor point act as a local home agent that tie up RCoA of mobile node to its LCoA. Mobile node need to send its Local binding update after get renewal of RCoA and LCoA to the MAP in order to establish binding between the RCoA and LCoA. Binding update should be send after mobile node received Binding Acknowledgement from the MAP to register the RCoA with Correspondent Nodes and Home Agent as mentioned in [2].

\section{II.2. Transmission Control Protocol (TCP)}

The state of network path determine window size of TCP congestion control mechanism dynamically. Abed et al in [5] explained that according to bandwidth availability in an end to end connection, data rate of TCP could be adjust by its congestion control although TCP has critical design. However, TCP has slow response in large congestion window so that TCP provides less performance in channel with high bandwidth. When segement transmission done and Acknowledgment (ACK) are sent by TCP receiver but it did not received by $\mathrm{TCP}$ sender, then connection become timeout where the first phase of start transmission called slow-start. Parallel to round trip time (RTT) which increase, the window size of TCP and the sending packet go up exponentially in slow start phase.

\section{II.3. High Speed Transmission Control Protocol (HS-TCP)}

High Speed Transmission Control Protocol (HSTCP) is a transport protocol that introduce new method by improving general algorithm of TCP's Additive Increase Multiplicative Decrease (AIMD. in reducing time of loss recovery. As provided in [5] by G. A. Abed and M. I. K. Jumari, large congestion windows could be support effectively by HS-TCP. AIMD algorithm will be used when congestion windows under the threshold while congestion windows more than threshold high speed AIMD algorithm will be chosen. The response distinction surroundings of the low congestion occurring in bottleneck could specify design of HS-TCP. This design also depend on response activation of standard TCP in packet loss rates environment

High Speed TCP is encouraged by some of the requirements below:

- When slow start condition appear, throughput could reach high point 
- Multiple retransmit timeout easily be recovered with less delay but high throughput.

- Additional support and feedback for router are not required.

- No additional feedback required from TCP receivers.

- The unrealistic rate of low packet loss did not needed while high throughut each connection could be achieved.

- In environments with moderate or high congestion HSTCP minimum performance are same with standard TCP.

- Performance of transient easily accepted, in term of congestion window rise in single round-trip time, reaction to advanced congetion and convergence times to fairness.

\section{Proposed Topology and Simulation}

Simulation has been created using discrete event simulator. This simulator are related to simulate network and communication analysis. Network simulator has ability to support environment to propose many kind of protocols such as mobile network, traffic and handover mobile device, muticast protocol. NS-2 also support wired and wireless channel condition. Although other simulators are widely available, ns-2 remains popular as it is freely available, the source is available for inspection and modification, and there is a large user community that provides contributions in the form of extensions that allow new protocols and systems to be simulated.

\section{III.1. Proposed Topology}

As explained before, the simulation used discrete event simulator namely Network Simulator to create proposed system and topology of HMIPv6. This simulator also used in order to monitor the behavior of High speed TCP and standard TCP because NS2 is better in providing TCP protocol simulation.

In this research, NS-2 version 2.31 used with ns2.31_fhmip.patch and this version installed over Linux Ubuntu 12.04 LTS. This topology varies depending on:

1. System is configured using bottleneck bandwidth $(\mathrm{Bw})$ which has different value in every simulation. The number will be increased start from $100 \mathrm{MB}, 500 \mathrm{MB}, 1 \mathrm{~GB}, 10 \mathrm{~GB}, 40 \mathrm{~GB}$, to
100GB.

2. The delay (D) values are 0 and $100 \mathrm{~ms}$.

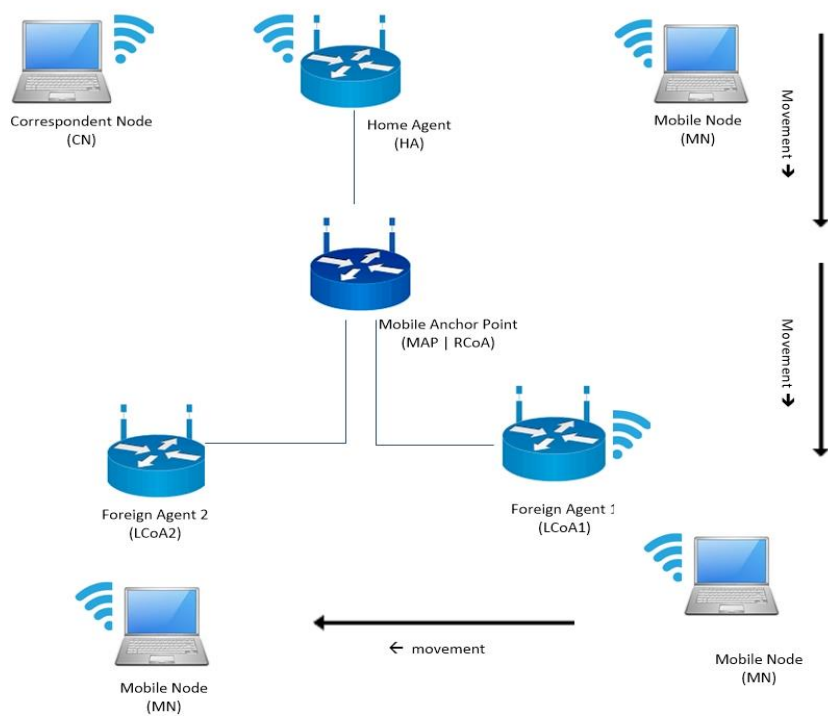

Fig. 2. Proposed topology of HMIPv6 Simulation

\section{III.2. Proposed Scenario}

This section describes the experiments performed to support this project. This experiment goal is provide performance comparison between TCP and HS-TCP. The considered scenario can be observed from Figure 2 above. The figure show a hierarchical topology of mobile internet protocol version 6 network.

Topology consists of one home agent have wireless and wired connection and it connected directly with mobile anchor point using wire. A Correspondent node perform indirect communication to mobile node via wireless attached to home agent. Between mobility anchor point and two access routers which both act as foreign agent connected by wire. Meanwhile, mobile node move from home agent to first foreign agent then goes to the next foreign agent via wireless communication and at the same time mobile node also communicate with its correspondent node.

In the topology, there are two link characteristics namely delay (milliseconds) and bandwidth (megabits/s). Two access routers FA1 (LCoA1) and FA2 (LCoA2) are set to 160 meters apart with free space in between. Coverage area of radio wave at 
every base station are 100 meters. Mobile node is initially locatesd near home agent, then it migrate towards first Local carrier of address and second Local carrier of address after simulation started. Mobile node move constanly with speed value is $15 \mathrm{~m} / \mathrm{s}$. A single simulation run is 20 seconds in duration studied here.

The implementation of HS-TCP is controlled by adjustment congestion control. This kind of TCP variant adapting to change of response function and the effect will appear when high congestion window happen. In this simulation TCP source and destination agent are created because it needed to proposes test behavior of congestion window.

TABLE I

SIMULATION PARAMETERS

\begin{tabular}{ll}
\multicolumn{2}{c}{ SIMULATION PARAMETERS } \\
\hline \multicolumn{1}{c}{ Parameter } & Value \\
\hline Transport protocols & TCP, \\
& HS-TCP \\
\hline The size of packet & 1500 Bytes \\
\hline The size of window & $100 \mathrm{Kbytes}$ \\
\hline Delay propagation of all links & $50 \mathrm{msec}$ \\
\hline User equipment link bandwidth & $1 \mathrm{Mbps}$ \\
\hline GW link bandwidth & $10 \mathrm{Mbps}$ \\
\hline Node link bandwidth & $100 \mathrm{Mbps}$ \\
\hline The time of simulation & $25 \mathrm{sec}$ \\
\hline
\end{tabular}

\section{Result and Analysis}

Topology network consists of two mobile nodes called Mobile Node (MN) and Correspondent Node $(\mathrm{CN})$. A full duplex-link is established between the all of nodes/routers using the given Bw and Tp. A queue limit is then computed for each of the routers. Normally, we will get an ack from a receiver within $2 \mathrm{Tp}$. In an ideal world, we want to keep the pipe full, so we would like $2^{*} \mathrm{Bw} * \mathrm{Tp}$ in the network at any point in time. To size the queue, we need to make it equal to the amount of data that can be in the network. The bandwidth-delay product is used to determine this value. Multiplying the $\mathrm{Bw}$ by the Tp indicates how many bits are stored on the wires, and multiplying by 2 gives a good queue size. In ns, queues are in units of packets, so we computed $2 * \mathrm{Bw}^{*} \mathrm{Tp}$ and converted the number into packets. This value was used to set the queue size for each router.

Once the topology has been created, ftp agents are created for each connection using the appropriate start times and the ns simulation is set to begin, running for 100 seconds. If HS-TCP is being tested, the windowOption is set as follows to indicate a HS-TCP connection rather than TCP.

\section{Agent/TCP set windowOption_ 8}

Once the simulation has completed, the tcl code will generate a trace file that contains the throughput of all runs. The throughput is computed as follows:

- Determine the number of packets that were sent over 100 seconds.

- Determine the number of bits sent. We assume the default packet size is 1000 bytes, and we know that 1 byte contains 8 bits. Therefore, multiplying the result of (1) by 1000 and by 8 will determine the number of bits successfully sent.

- Finally, to determine the throughput, divide the result of (2) by the amount of time that elapsed from the start of the ftp agent until the simulation ended.

Running program will read files generated by ns/tcl script and compute the average throughput of all senders. The throughput of the network is the amount of data that traversed the network successfully per unit time in Kbits (Kilobits). For each run, we measure the throughput for each TCP connection and take their average. If delay $(\mathrm{D})=0$, then it makes sense to average the throughput of all tcp connections and end up with one average throughput. This can be done because the only differences in the tcp connections are the start times. Computing the average throughput where the delay $(D)=100$, however, is not as simple. We must compute the average throughput of each connection rather than the average throughput of all tcp connections. Averaging the throughput of all tcp connections would give misleading results since the delay of each tcp connection differs by at least $100 \mathrm{~ms}$.

Information below are the output example:

$\begin{array}{llll}336990.09 & 340543.69 & 337113.36 & 337803.35 \\ 339167.92 & 340461.49 & 337031.17 & 337232.93 \\ 338601.04 & 339351.63 & 337864.94 & 341013.73 \\ 337583.31 & 336976.39340283 .50338375 .69\end{array}$


$\begin{array}{lllll}339409.50 & 340201.42 & 336894.27 & 337461.55\end{array}$ $\begin{array}{llll}338950.45 & 339958.60 & 336812.18 & 336892.29\end{array}$ $\begin{array}{llll}338384.29 & 339011.58 & 337646.03 & 340917.56\end{array}$ 337487.16338420 .33338331 .07339205 .33

From this output, we can tell that the throughput of the 1st run of the tcp connection between sender1 and destination1 was 336990.09 bps, the throughput of the 2 nd run of the tcp connection between sender1 and destination 1 was 340543.69 bps.

\section{IV.1. Bandwidth Configuration : $100 \mathrm{Mbps}$ between routers}

Simulation result displayes thath high speed TCP had throughput 32\% higher than TCP when environment set up delay of propagation with value $50 \mathrm{~ms}$ as shown at fig. 3 .

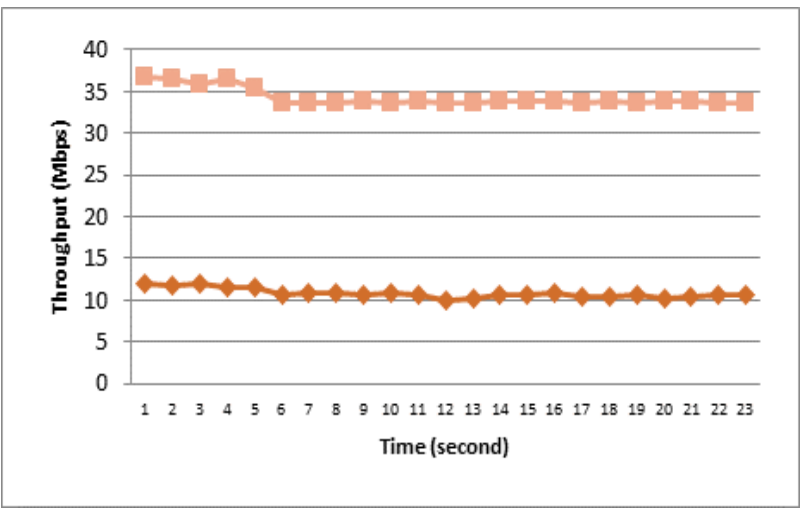

Fig. 3. Throughput when bandwidth is $100 \mathrm{Mbps}$

\section{IV.2. Bandwidth Configuration : $500 \mathrm{Mbps}$ between routers}

Topology link is configured with propagation delay value $50 \mathrm{~ms}$, then the results indicated that HS-TCP had a quite higher throughput of $96 \%$ than the bandwidth configurations. The outcome of this simulation system depicted in Fig.4 below.

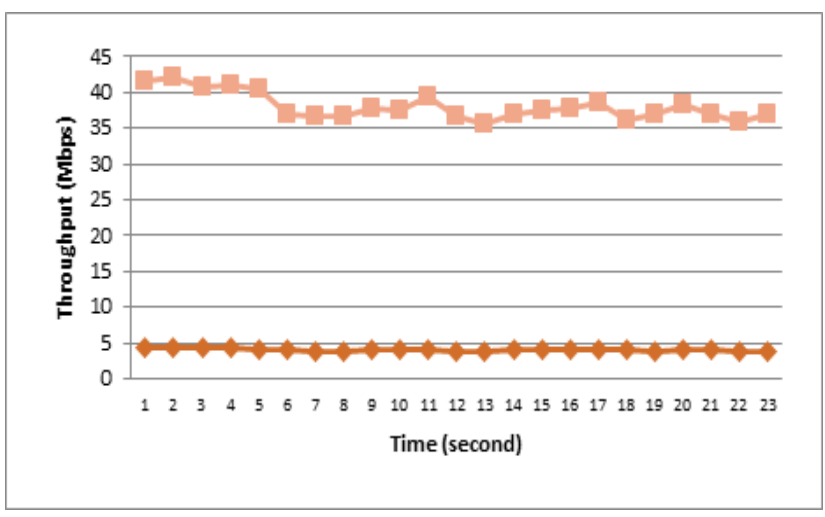

Fig. 4. Throughput when using bandwidth $500 \mathrm{Mbps}$

\section{IV.3. Bandwidth Configuration: 1 Gbps between routers}

The value of HSTCP throughput reached $85 \%$ higher compared to TCP when propagation delay of system is set to $50 \mathrm{~ms}$ and the results showed at fig.5.

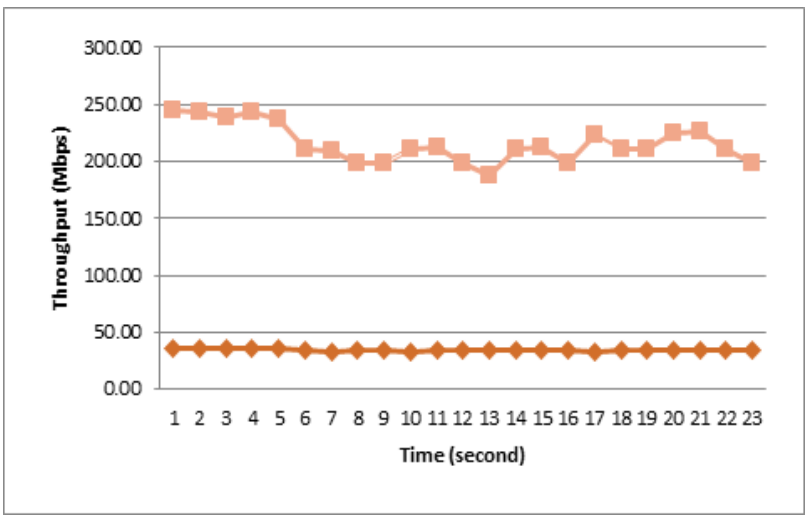

Fig. 5. Throughput when using bandwidth $1 \mathrm{Gbps}$

IV.4. Bandwidth Configuration: 10 Gbps between routers

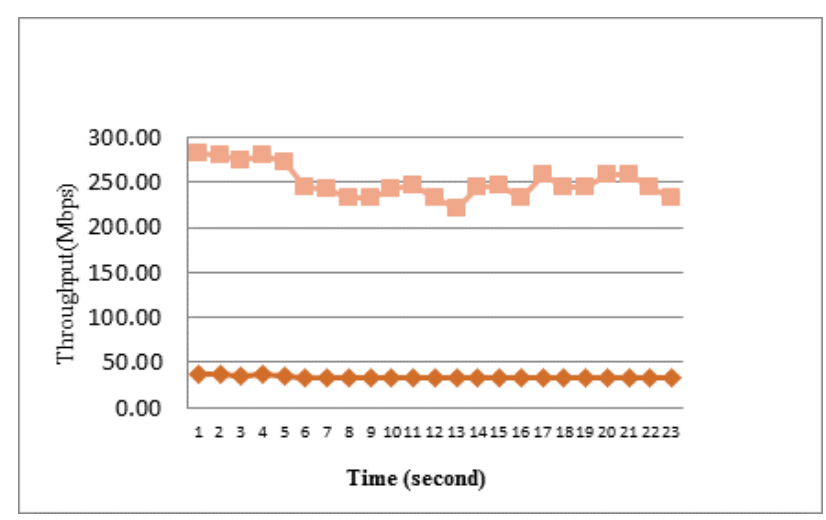

Fig. 6. Throughput when using bandwidth $10 \mathrm{Gbps}$ 
High speed TCP reveals the result that it had $86 \%$ higher throughput than TCP in high speed bandwidth configuration. This simulation has similar outcome under 1 and 10 Gbps bandwidth link between nodes.

\section{Conclusion}

Based on the data simulation, the result showed that compared to TCP high bandwidth, HS-TCP with high bandwidth has higher throughput.This information could be conclude that HS-TCP has a better performance than TCP by looking at the throughput which run on the HMIPv6 topology.

This simulation also proved that HS-TCP are suitable to be implemented in neighborhood supporting high speed. With bandwidth at 100 Mbps, we can see that HS-TCP is $32 \%$ better than HS-TCP, in $500 \mathrm{Mbps}$ HS-TCP is better at $96 \%$ than TCP. In 1Gbps, HS-TCP is better at $85 \%$ than TCP and in 10Gbps, HS-TCP is better at $86 \%$ than TCP.

\section{Acknowledgements}

This work was supported by Universitas Muhammadiyah Yogyakarta and Universitas Indonesia.

\section{References}

[1] H. Bo, C. Shanzhi, J.Xiaoyan, "A Performance Evaluation of IP Mobility Support Protocols". Second International Conference on MultiMedia and Information Technology, 2010.

[2]Soliman et al, "Hierarchical Mobile IPv6 (HMIPv6) Mobility Management" Request For Comments: 5380, 2008.

[3] K. Zulkeflee, S. Z. Mohamad, "The Future Enhancements of Mobile IPv6 Micro-Mobility Management". Second International Conference on Network Applications, Protocols and Services, 2010.

[4] Z. Kusin1, M. S. Zakaria, "Dynamic Load Control Mechanism in Hierarchical MIPv6", ICEEI, 17-19 July, Bandung, Indonesia, 2011

[5] G. A. Abed, M. I. K. Jumari. "A Comparison and Analysis of Congestion Window for HS-TCP, FullTCP, and TCP-Linux in Long Term Evolution System Model". ICOS, 25 - 28 September, Langkawi, Malaysia. 2011.

\section{Authors' information}

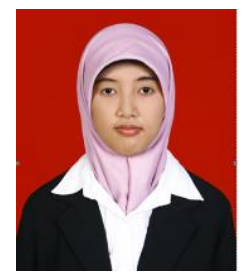

Nur Hayati currently is a lecturer and researcher in Universitas Muhammadiyah Yogyakarta. She graduated from telecommunication engineering major in EEPIS-ITS Surabaya and continued her master degree in electrical engineering major Universitas Indonesia Depok.

The author previous paper with her team are concern about security. Paper with title "Dual Key Triple Encryption Text Based Message Using Cryptography And Steganography" is published on IJTA, 2013.

Nur Hayati, S.ST. M.T. is member of senior instructor in Cisco Networking Academy Program and Unity of Indonesian Engineers (Persatuan Insinyur Inndonesia) 